\title{
Perinatal arterial ischemic stroke and multiple thrombo-emboli in a term newborn
}

\author{
Sani Bukari ${ }^{1}$, Abdul Hafeez Siddiqui ${ }^{2}$, Madhvi Rajpurkar ${ }^{1}$, Meera Chitlur ${ }^{1}$ \\ ${ }^{1}$ Division of Hematology/Oncology, Department of Pediatrics, Children's Hospital of Michigan, Wayne State University, Detroit, USA \\ ${ }^{2}$ Division of Hematology/Oncology, Department of Pediatrics, Children's and Women's Hospital, University of South Alabama, \\ Mobile, USA \\ Email: mchitlur@med.wayne.edu
}

Received 3 July 2011; revised 27 October 2011; accepted 21 November 2011

\begin{abstract}
The etiology of perinatal arterial ischemic strokes may be maternal, fetal or both. Venous thrombo-embolism is almost always catheter associated in newborns. Preliminary guidelines for management of perinatal arterial ischemic strokes exist but are mostly based on adult studies. We report a unique case of perinatal arterial ischemic stroke, lower extremity arterial and inferior vena-caval thrombosis. Our patient was treated with unfractionated heparin for 10 days then switched to low molecular heparin to complete 6 months of therapy. The patient responded well to therapy and shows minimal signs of permanent neurologic deficits after 6 months.
\end{abstract}

Keywords: Perinatal Arterial Ischemic Strokes; Heparin

\section{INTRODUCTION}

Thrombosis is a rare but important clinical entity in children. Perinatal arterial ischemic stroke is defined as arterial thrombosis or embolization leading to focal disruption of cerebral blood flow, after 20 weeks of gestation through 28 days after birth [1,2]. Among the pediatric population, neonates have the highest incidence of arterial ischemic strokes, reported to be as high as 18 per 100,000 full term, live births [3]. The incidence is reported to be slightly higher in black male infants compared to other ethnicities. The left middle cerebral artery and left cerebral hemisphere is the most common territory involved, the reason for which is not clear. Risk factors of perinatal arterial ischemic strokes are either maternal or fetal although in majority of the cases, no clinical risk factor is identified [4]. Clinical presentation is nonspecific, ranging from abnormal neurologic exam and seizures to hemiparesis or hemiplegia. Preliminary guidelines for management of perinatal ischemic strokes exist and are mostly based on adult studies [5]. Venous thrombo-embolism in children on the other hand has an extremely low reported annual incidence of around $1.4 / 100,000$ and is almost always catheter associated [6].

We present a case of a full term newborn with multiple arterial and venous emboli possibly secondary to placental vascular thrombosis. She responded well to heparin therapy without thrombolytic interventions and had minimal neurologic deficit at six month follow up

\section{CASE REPORT}

An African American female was born to a 19 year old primi-para mother at 41 weeks of gestation. Mother had no significant personal or family history of medical illness or coagulation disorders. She received good prenatal care with a negative screening for sexually transmitted disease. She was found to be colonized with group B streptococcus and received two doses of vancomycin prior to delivery. Prenatal ultrasound studies were within normal limits and did not suggest any congenital anomalies. The baby was born via uncomplicated vaginal delivery with normal APGARs and vital signs. Anthropometric measurements were appropriate for gestational age. Upon physical examination, immediately after delivery, the baby was noted to have pallor of the right lower extremity with absent femoral and dorsalis pedis artery pulses on the same side. A Doppler ultrasound evaluation performed within the first hour of birth showed a thrombus in the right external iliac artery extending distally to the popliteal artery occluding the vessels. She was also found to have a thrombus in the inferior vena-cava. She was transferred to the neonatal intensive care unit (NICU) for further management. In the NICU, the baby was clinically stable with no neurologic deficits. A screening head ultrasound, performed at hour 6 of birth, suggested a small germinal matrix hemorrhage. This was followed by a magnetic resonance imaging (MRI), magnetic resonance arteriography (MRA) and venography (MRV) of the whole body around hour 10 of delivery. There was again a low T2 signal structure seen at the expected position in the inferior vena cava (IVC) 
at the level of right hepatic lobe with non-visualization of IVC caudal to this position (Figure 1). Extensive collateral vessels were visualized around the obliterated segment of the IVC, indicating either a thrombosed or congenitally absent IVC. There was also suggestion of a left adrenal gland hemorrhage. On the MRI scans of the brain, a large acute infarct involving the left middle cerebral artery territory was reported, associated with complete occlusion of the distal segment of supraglenoid internal carotid artery as well as middle cerebral artery (Figure 2). There was also a small embolic infarct on the right frontal lobe. Further investigation including an echocardiogram, renal ultrasound and electroencephalo-

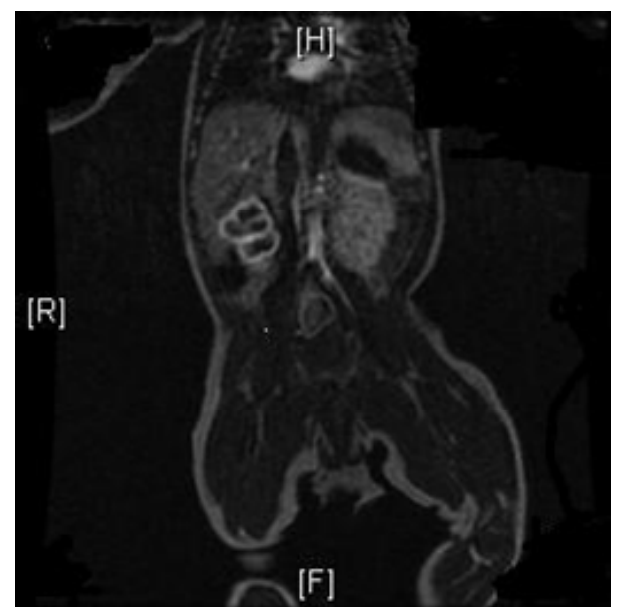

Figure 1. MRA/MRV showing attenuation in flow in the inferior vena cava just below the right hemi diaphragm and attenuation of flow in the right external iliac artery both secondary to thrombi.

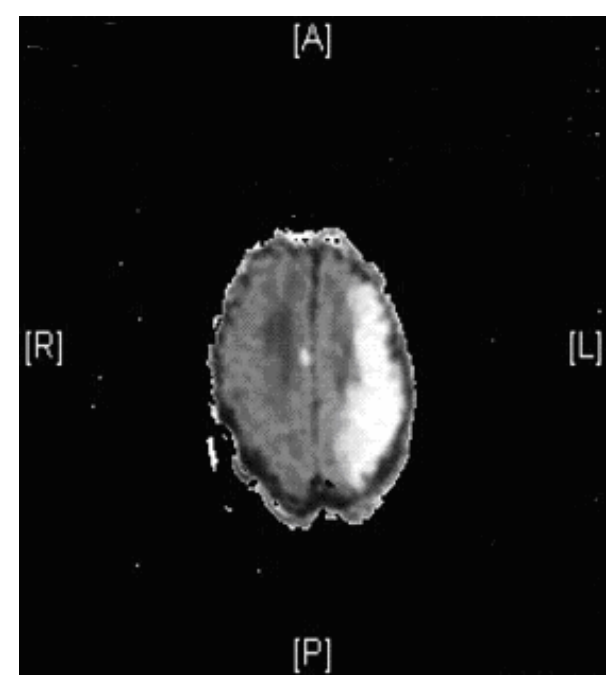

Figure 2. MRI of the brain showing large infarct involving left parietal, temporal and occipital lobes corresponding to left middle cerebral artery territory. gram did not show any abnormalities.

Thromboembolic evaluation, including factor II, factor VIII, antithrombin III, protein S and protein C activity were all within normal limit for age. Genetic testing of both baby and the mother is negative for mutations for factor V Leiden, prothombin variant or methylene tetrahydrofolate reductase. Mother had a borderline low protein C activity at 64\% (normal range: 70\% - 128\%). Since this was done at immediate post-partum period, she was advised to follow up with adult hematologist. The placental histopathology revealed fetal thrombotic vasculopathy, suggesting possible intrauterine thrombotic events. The baby was immediately started on low dose heparin infusion at $15 \mathrm{Units} / \mathrm{Kg} / \mathrm{hr}$ and was switched to enoxaparin after 10 days of therapy. The dose of enoxaparin was adjusted to bring the activated factor IX levels within the therapeutic range. No thrombolytic intervention was recommended due to a small germinal matrix hemorrhage seen on head ultrasound. She responded well to the therapy, with no evidence of clot propagation, worsening of intracerebral hemorrhage and with significant improvement of perfusion of left lower extremity within the first day of life hence did not require surgical intervention. Serial imaging studies showed gradual return of blood flow through the IVC and stable cerebral vasculature. She was discharged home on enoxaparin for 6 months with close outpatient follow-up. Repeat MRI, MRA and MRV imaging at 5 months age showed resolution of inferior vena-cava clot and brain changes consistent with her chronic infarcts. She is receiving physical therapy and has a minimal residual right sided weakness. She continues to thrive and has achieved age appropriate developmental milestones. She continues to follow-up with her neurologist and is expected to do well if affected cerebral hemisphere is non-dominant.

\section{DISCUSSION}

This is a unique case of perinatal arterial ischemic stroke along with lower extremity arterial and IVC thrombosis in a term baby. The etiology of perinatal arterial ischemic stroke is multifactorial and is not identified in most cases $[7,8]$. Maternal and fetal factors may interplay in the pathogenesis. Normal physiology of pregnancy put mothers in a hypercoagulable state, hence a slightly lower protein $C$ level in our patient's mother. Other factors in maternal history which have been associated with perinatal arterial ischemic strokes include history of infertility, chorioamnionitis, premature rupture of membrane, placental vasculopathy and preeclampsia. Congenital heart diseases, coagulopathy, infections, trauma, drugs, perinatal asphyxia and intravascular catheters constitute some of the fetal risk factors $[7,9]$. 
Our patient was symptomatic at birth and we postulate that she probably had an in-utero triggering event. Histopathology of the placenta revealed thrombotic vasculopathy which could be the initial triggering event leading to thrombosis in the IVC in-utero. This ultimately resulted in obliteration and fibrosis of a segment of the IVC with establishment of extensive collateral circulation which help venous return from lower extremities. We also postulate subsequently that, a dislodged thrombus from IVC-thrombus passed through the patent foramen ovale to the left heart and hence to systemic circulation (middle cerebral and external iliac arteries), resulting in the stroke and the right lower extremity ischemia. Whilst chorioamnionitis has been identified as an important risk factor of placental vasculopathy, the exact etiology in our patient is not completely clear.

There is no consensus on the treatment of perinatal ischemic strokes. The American College of Chest Physicians (ACCP) recommends administration of heparin (unfractionated or low-molecular weight) as first line therapy for symptomatic patients [10]. Since Recurrence of symptomatic thromboembolism in children with history of perinatal arterial ischemic strokes in one study was only $3.3 \%$ [11], long term use of antiplatelet or anticoagulation therapy is not recommended until a recurrence. On the other hand the American Heart Association does not recommend any treatment for perinatal arterial ischemic strokes [9]. According to the Canadian Pediatric Ischemic Stroke Registry, most of the neonates that were treated received low molecular weight heparin and none of them were reported to have hemorrhagic complications associated with deaths or neurologic deficits [12]. Our patient was treated with unfractionated heparin for 10 days followed low molecular weight heparin, for duration of 6 months. The germinal matrix bleed is a contraindication to thrombolytic therapy [13]. Unfractionated heparin was used since anticoagulation can easily be reversed in the event of a major bleed. She responded well to therapy with re-establishment of perfusion in the right lower limb.

Some studies suggest, about a third of neonates with arterial ischemic strokes develops permanent neurologic deficits or cerebral palsies [5]. Stable intelligence quotient (IQ) and age appropriate language skills have been reported [14]. Nevertheless long term neurologic followup is crucial. Our patient continues to do well on physical therapy without signs of significant permanent neurologic damage. There is no other risk factor for thrombosis since the contact with placenta is no longer present after birth.

In conclusion, most of the time, no risk factors are identified in perinatal arterial ischemic strokes; therefore, Physicians do not tend to treat them. However, if the patient's condition warrants an immediate intervention, unfractionated heparin should be considered as a first line therapy. Also, the evaluation for thromboembolism in the perinatal period must include histopathology review of the placenta.

\section{REFERENCES}

[1] Dlamini, N. and Kirkham, F.J. (2009) Stroke and cerebrovascular disorders. Current Opinion in Pediatrics, 21, 751-761. doi:10.1097/MOP.0b013e328332c735

[2] Strater, R., Kurnik, K., Heller, C., Schobess, R., Luigs, P. and Nowak-Gottl, U. (2001) Aspirin versus low-dose low- molecular-weight heparin: Antithrombotic therapy in pediatric ischemic stroke patients: A prospective follow-up study. Stroke, 32, 2554-2558. doi:10.1161/hs1101.097379

[3] Bernard, T.J. and Goldenberg, N.A. (2010) Pediatric arterial ischemic stroke. Hematology/Oncology Clinics of North America, 24, 167-180. doi:10.1016/j.hoc.2009.11.007

[4] Amlie-Lefond, C., Sebire, G. and Fullerton, H.J. (2008) Recent developments in childhood arterial ischaemic stroke. Lancet Neurology, 7, 425-435. doi:10.1016/S1474-4422(08)70086-3

[5] Jordan, L.C., Rafay, M.F., Smith, S.E., Askalan, R., Zamel, K.M., DeVeber, G., et al. (2010) Antithrombotic treatment in neonatal cerebral sinovenous thrombosis: Results of the international pediatric stroke study. Journal of Pediatrics, 156, 704-710.

[6] Van Ommen, C.H., Heijboer, H., Buller, H.R., Hirasing, R.A., Heijmans, H.S. and Peters, M. (2001) Venous thromboembolism in childhood: A prospective two-year registry in the Netherlands. Journal of Pediatrics, 139, 676-681. doi:10.1067/mpd.2001.118192

[7] Pavlakis, S.G. and Levinson, K. (2009) Arterial ischemic stroke: Common risk factors in newborns and children. Stroke, 40, S79-S81.

doi:10.1161/STROKEAHA.108.531749

[8] Raju, T.N., Nelson, K.B., Ferriero, D. and Lynch, J.K. (2007) Ischemic perinatal stroke: Summary of a workshop sponsored by the National Institute of Child Health and Human Development and the National Institute of Neurological Disorders and Stroke. Pediatrics, 120, 609616. doi:10.1542/peds.2007-0336

[9] Roach, E.S., Golomb, M.R., Adams, R., Biller, J., Daniels, S., Deveber, G., et al. (2008) Management of stroke in infants and children: A scientific statement from a Special Writing Group of the American Heart Association Stroke Council and the Council on Cardiovascular Disease in the Young. Stroke, 39, 2644-2691. doi:10.1161/STROKEAHA.108.189696

[10] Monagle, P., Chalmers, E., Chan, A., DeVeber, G., Kirkham, F., Massicotte, P., et al. (2008) Antithrombotic therapy in neonates and children. Chest, 133, 887S-968S. doi:10.1378/chest.08-0762

[11] Kurnik, K., Kosch, A., Strater, R., Schobess, R., Heller, C. and Nowak-Gottl, U. (2003) Recurrent thromboembolism in infants and children suffering from symptomatic neo- 
natal arterial stroke: A prospective follow-up study. Stroke, 34, 2887-2892.

doi:10.1161/01.STR.0000103745.03393.39

[12] DeVeber, G., Andrew, M., Adams, C., Bjornson, B., Booth, F., Buckley, D.J., et al. (2001) Cerebral sinovenous thrombosis in children. The New England Journal of Medicine, 345, 417-423. doi:10.1056/NEJM200108093450604

[13] Manco-Johnson, M.J., Grabowski, E.F., Hellgreen, M., Kemahli, A.S., Massicotte, M.P., Muntean, W., et al. (2002) Recommendations for tPA thrombolysis in children. On behalf of the Scientific Subcommittee on Perinatal and Pediatric Thrombosis of the Scientific and Standardization Committee of the International Society of Thrombosis and Haemostasis. Thrombosis and Haemostasis, 88, 157158.

[14] Ballantyne, A.O., Spilkin, A.M. and Trauner, D.A. (2007) Language outcome after perinatal stroke: Does side matter? Child Neuropsychology, 13, 494-509. doi:10.1080/09297040601114878 